\title{
Feasibility and Outcomes of Multivisceral Resection in Locally Advanced Colorectal Cancer: Experience of a Tertiary Cancer Center in North-East India
}

\author{
Joydeep Purkayastha, Pritesh Rajeev Singh, Abhijit Talukdar, Gaurav Das, Jitin Yadav, Srinivas Bannoth \\ Department of Surgical Oncology, Dr. B. Borooah Cancer Institute, Guwahati, India
}

Purpose: Locally advanced colorectal cancer may require an en bloc resection of surrounding organs or structures to achieve complete tumor removal. This decision must weigh the risk of complications of multivisceral resection against the potential survival benefit. The purpose of this study is to review a single-center experience of feasibility of en bloc multivisceral resections for locally advanced colorectal carcinoma and to examine the effect of surgical experience on immediate outcome and rate of R0 resections.

Methods: This is a study of 27 patients who underwent multivisceral resection for locally advanced colorectal carcinoma which was performed at our institute from January 2016 to December 2019. Among the 27 patients aged between 21 and 76 years (mean age, $48.67 \pm 7.3$ years), 13 were males and 14 were females. Overall 18 patients had primary colon carcinoma and 9 had primary rectal carcinoma. All rectal cancer patients received neoadjuvant chemoradiation. All patients underwent surgery with curative intent. All patients underwent open surgery of which $66.7 \%$ underwent colectomy, $14.8 \%$ underwent anterior resection, $11.1 \%$ underwent Miles procedure, and 7.4\% underwent pelvic exenteration.

Results: The mean operative time was $268.14 \pm 72.2$ minutes and the median amount of blood units transfused was 2.07 units. The mean hospital stay was $13.67 \pm 3.4$ days. Histologically, $44.4 \%$ of patients had well-differentiated adenocarcinoma and 55.6\% had moderately differentiated adenocarcinoma. The final histopathological examinatio $\mathrm{n}$ revealed malignant infiltration of the adjacent organs in 19/27 patients (70.4\%). Pathological complete response was seen in 2 patients. R0 resection rate achieved was $96.3 \%$. Lymph node metastasis was seen in $66.7 \%$ of patients with colon cancer and $11.1 \%$ with rectal cancer with overall mean number of harvested lymph nodes being $12.44 \pm 3.01$. Postoperative complications were identified in 7 patients (25.9\%), while mortality was seen in 2 (7.4\%).

Conclusion: Multivisceral resection for advanced colorectal cancer invading into the adjacent organ may be performed with acceptable morbidity and mortality.

Keywords: Colorectal neoplasms; Multivisceral resection; Surgical resection

\section{INTRODUCTION}

Globally, colorectal cancer accounts for approximately $10 \%$ of all

Received: Mar 13, 2020 - Revised: May 18, 2020 - Accepted: Jun 3, 2020 Correspondence to: Pritesh Rajeev Singh, M.Ch.

Department of Surgical Oncology, Dr. B. Borooah Cancer Institute, AK Azad Road, Gopinath Nagar Rd, Bishnu Rabha Nagar, Guwahati, Assam 781016, India Tel: +91-99695-34490

E-mail: singhpritesh17@gmail.com

ORCID: https://orcid.org/0000-0003-4965-936X

(C) 2021 The Korean Society of Coloproctology

This is an open-access article distributed under the terms of the Creative Commons Attribution NonCommercial License (https://creativecommons.org/licenses/by-nc/4.0) which permits unrestricted noncommercial use, distribution, and reproduction in any medium, provided the original work is properly cited. new cancers [1]. Colorectal cancer invading into the adjacent organs/structures is detected in $5 \%$ to $20 \%$ of all surgical interventions performed for the management of colorectal cancer [2]. These adhesions may be either due to frank tumor infiltration or due to peritumoral inflammation. However, the nature of these adhesions cannot be ascertained intraoperatively. Therefore, the standard management entails en bloc resection of the diseased organ along with adjacent organ infiltration. Neoadjuvant treatment with chemotherapy, radiation, or a combination of both can significantly lead to downsizing of the disease thereby facilitating resection of the tumor with safe radial and circumferential margins. We share our experience regarding the feasibility of en bloc multivisceral resection for advanced colorectal cancer, the immediate 
surgical outcomes in regards to morbidity and mortality, and the proportion of R0 resections.

\section{METHODS}

This retrospective study was approved by the Institutional Review Board of Dr. B. Borooah Cancer Institute (BBCI-TMC/Misc-119/ MEC/282/2019). Written informed consent was obtained for publication of this study and accompanying images.

Twenty-seven patients underwent multivisceral resection for locally advanced colorectal carcinoma at our institute from January 2016 to December 2019. Among the 27 patients aged between 21 and 76 years (mean age, $48.67 \pm 7.3$ years), 13 were males and 14 were females.

Pretreatment staging was done by taking a detailed history and conducting a physical examination in the outdoor office. Pretreatment complete blood tests and carcinoembryonic antigen (CEA) levels were obtained. Full colonoscopy was performed in all patients.

Patients with colonic and upper rectal primary underwent contrast-enhanced computed tomography (CECT) of the abdomen and thorax. Those with disease in the mid rectum, lower rectum, and anorectum underwent magnetic resonance imaging (MRI) of the pelvis and CECT of the abdomen and thorax. Patients in whom imaging suggested stomach infiltration underwent esophagogastroduodenoscopy. Four patients with obstructive symptoms underwent fecal diversion before commencing the treatment. The rectal cancer patients received preoperative chemoradiation with capecitabine and $50.4 \mathrm{~Gy}$ in 28 fractions. They were reevaluated with MRI 6 to 8 weeks later. One patient who achieved significant symptomatic relief after chemoradiation defaulted for 2.5 years. One patient with mid-rectal tumor received 4 cycles of chemotherapy (5-fluorouracil + oxaliplatin) after radiation when the radiological circumferential margin was deemed to be positive. All patients with colonic primary were taken up for upfront surgery.

CEA levels were elevated ( $>3 \mathrm{ng} / \mathrm{mL}$ ) in $77.8 \%$ patients ( 21 of 27). All patients underwent surgery with curative intent. All patients underwent open surgery of which $66.7 \%$ underwent colectomy, $14.8 \%$ underwent anterior resection, $11.1 \%$ underwent Miles procedure, and $7.4 \%$ underwent pelvic exenteration.

The criteria for multivisceral resection was based on imaging features; mainly bulky disease in the primary with loss of fat planes with the adjacent or surrounding structures or organs or frank infiltration. Multivisceral resection was preplanned in all patients. Intraoperatively, multivisceral resection was performed when the tumor-bearing part could not be separated from the surrounding structures or organs without compromising the oncologic outcomes.

Colectomy: Resection of the tumor-bearing colon with longitudinal margins of at least $5 \mathrm{~cm}$ on either side, the corresponding mesocolic tissue along with a D3 lymphadenectomy (i.e., remov- ing the nodal tissue along the lateral part of the superior mesenteric artery).

Anterior resection: For tumors of the rectosigmoid junction, upper and middle rectum, removal of the rectum with at least a $5-\mathrm{cm}$ margin proximally and 2-cm margin distally, total mesorectal excision, with high ligation of the inferior mesenteric artery.

Miles procedure (proctosigmoidectomy): For low-rectal and anorectal tumors when sphincter salvage is not possible, total mesorectal excision, high ligation of the inferior mesenteric artery, and a permanent end sigmoid colostomy.

Pelvic exenteration: Removal of all the pelvic organs in females with permanent fecal and urinary diversions (i.e., anorectum, urinary bladder, and uterus).

\section{RESULTS}

The mean operative time was $268.14 \pm 72.2$ minutes and the median amount of blood units transfused was 2.07 units. The mean hospital stay was $13.67 \pm 3.4$ days. Histologically, $44.4 \%$ of patients had well-differentiated adenocarcinoma and $55.6 \%$ had moderately differentiated adenocarcinoma. The final histopathological examination revealed malignant infiltration of the adjacent organs in 19 of 27 patients (70.4\%). R0 resection rate achieved was $96.3 \%$. Lymph node metastasis was seen in $66.7 \%$ of patients with colon cancer and $11.1 \%$ with rectal cancer with overall mean number of harvested lymph nodes being $12.44 \pm 3.01$. Postoperative complications were identified in 7 patients (25.9\%), while mortality was seen in 2 patients (7.4\%). The demographic details, location of tumor, pretreatment CEA levels, American Society of Anesthesiologists physical status classification, complications, postoperative hospital stay, and postoperative histopathology information is shown in Table 1.

We obtained pathologic complete response in 2 patients of rectal cancer after chemoradiation. The first was a case of mid-rectal tumor with urinary bladder infiltration. The patient received capecitabine based long-course chemoradiation. MRI after chemoradiation and before surgery showed dense adhesion of the disease site with urinary bladder and the uterus. The patients underwent pelvic exenteration, i.e., removal of urinary bladder and uterus with bilateral adnexa in addition to proctectomy. The second was also a mid-rectal tumor in a postmenopausal lady with infiltration into the uterus. The patient received capecitabine based longcourse chemoradiation. MRI after chemoradiation and before surgery showed persistent adhesion between the disease site and the uterus. The patient underwent hysterectomy and bilateral salpingo-oophorectomy in addition to proctectomy. In both the cases, it was difficult to ascertain on imaging and even intraoperatively whether the adhesion was due to persistent disease or radiation-induced fibrosis.

Preoperatively the patients were counseled accordingly and informed consent was obtained to go ahead with multivisceral resection. Since all the patients in our study were in stage III, they 


\section{Coloproctology Joydeep Purkayastha, et al.}

Table 1. Demographic and clinical details of the participants

\begin{tabular}{|c|c|c|c|c|c|c|c|c|c|c|c|}
\hline $\begin{array}{l}\text { Patient } \\
\text { No. }\end{array}$ & $\begin{array}{l}\text { Age } \\
\text { (yr) }\end{array}$ & Sex & Location of tumor & $\begin{array}{l}\text { CEA level } \\
\text { (ng/mL) }\end{array}$ & $\begin{array}{c}\text { ASA PS } \\
\text { classification }\end{array}$ & Complication & $\begin{array}{l}\text { Postoperative } \\
\text { stay }\end{array}$ & $\begin{array}{c}\text { CD } \\
\text { grade }\end{array}$ & P stage & Node & Tumor grade \\
\hline 1 & 60 & M & Ascending colon & 39 & $\|$ & None & 9 Days & I & T4bNO & 0 of 28 & Moderately differentiated \\
\hline 2 & 21 & $\mathrm{~F}$ & $\begin{array}{l}\text { Rectosigmoid } \\
\text { junction }\end{array}$ & 4.8 & I & None & 9 Days & I & T4bNO & 0 of 6 & Well differentiated \\
\hline 3 & 60 & M & Transverse colon & 2.4 & $\|$ & None & 14 Days & I & T4bNO & 0 of 12 & Moderately differentiated \\
\hline 4 & 47 & M & Ascending colon & 1.4 & I & None & 7 Days & I & T4bN1b & 2 of 23 & Well differentiated \\
\hline 6 & 42 & M & $\begin{array}{l}\text { Entire colon with } \\
\text { colonic polyps }\end{array}$ & $>400$ & I & Burst abdomen & 19 Days & $\| l l b$ & T4bN1c & 3 of 21 & Well differentiated \\
\hline 7 & 53 & M & Sigmoid colon & 14.1 & $\|$ & None & 9 Days & । & T4bN1b & 2 of 16 & Well differentiated \\
\hline 8 & 55 & $\mathrm{~F}$ & Descending colon & 0.8 & I & None & 13 Days & I & T4bN1a & 1 of 13 & Well differentiated \\
\hline 9 & 31 & $\mathrm{~F}$ & Anorectum & 3 & I & Bowel obstruction & 2 Months & Illb & T4bN1b & 2 of 15 & Moderately differentiated \\
\hline 11 & & & & & & & & & cT4bN2 & & \\
\hline 12 & 38 & M & Splenic flexure & 3 & I & None & 10 Days & । & T4bN1b & 2 of 14 & Well differentiated \\
\hline 13 & 63 & M & Transverse colon & 30.3 & $\|$ & Expired & & V & T4bN2b & 13 of 24 & Moderately differentiated \\
\hline \multirow[t]{2}{*}{14} & 49 & $\mathrm{~F}$ & Mid rectum & 2.4 & I & None & 9 Days & । & T2NO & 0 of 4 & Well differentiated \\
\hline & & & & & & & & & cT4bN1 & & \\
\hline \multirow[t]{2}{*}{15} & 47 & $\mathrm{~F}$ & Mid rectum & 218 & I & $\begin{array}{l}\text { Hematuria, urinary } \\
\text { retention, and } \\
\text { prolonged FC }\end{array}$ & $\begin{array}{l}16 \text { Days } \\
\text { (discharged } \\
\text { on catheter) }\end{array}$ & $\|$ & T3NO & 0 of 3 & Moderately differentiated \\
\hline & & & & & & & & & cT4bN1 & & \\
\hline 21 & 55 & M & Caecum & 10 & $\|$ & None & 8 Days & । & T4bN1b & 2 of 16 & Moderately differentiated \\
\hline 22 & 66 & M & Transverse colon & 34.2 & $\|$ & None & 10 Days & I & T4bNO & 0 of 15 & Well differentiated \\
\hline 23 & 64 & $\mathrm{~F}$ & Mid rectum & 2.6 & $\|$ & None & 11 Days & I & T3NO & 0 of 11 & Moderately differentiated \\
\hline 24 & 35 & $\mathrm{~F}$ & Hepatic flexure & 105 & I & None & 10 Days & I & T4bN1b & 2 of 15 & Moderately differentiated \\
\hline 25 & 50 & M & Sigmoid colon & 147 & । & None & 9 Days & I & T4bN1a & 1 of 14 & Moderately differentiated \\
\hline 26 & 36 & $\mathrm{~F}$ & Lower rectum & 0.3 & I & None & 10 Days & I & T4bNO & 0 of 2 & Moderately differentiated \\
\hline 27 & 42 & $M$ & Sigmoid colon & $>400$ & I & None & 10 Days & । & T4bNO & 0 of 6 & Well differentiated \\
\hline
\end{tabular}

CEA, carcinoembryonic antigen; ASA, American Society of Anesthesiologists; PS, physical status; CD, Clavien-Dindo classification; P stage, postoperative histopathology stage; M, male; F, female; pCR, pathologic complete response; FC, Foley catheterization.

received capecitabine and oxaliplatin adjuvant chemotherapy (except the 2 mortalities). Adjuvant therapy was decided based on clinical staging before chemoradiation.
The median follow-up period is 17 months (range, 2 to 61 months). There were 3 recurrences in the follow-up period; 2 lo$\mathrm{cal}$ and 1 systemic. Local recurrences were seen in the pelvic side- 
Table 2. The adjacent organs resected

\begin{tabular}{ll}
\hline Organ & No. \\
\hline Abdominal wall including musculature & 1 \\
Kidney+ureter & 1 \\
Liver segment & 2 \\
Spleen & 1 \\
Pancreas & 1 \\
Stomach & 2 \\
Small bowel & 6 \\
Large bowel & 2 \\
Vagina & 2 \\
Uterus+ovary & 8 \\
Urinary bladder & 6
\end{tabular}

wall in a rectal cancer patient who underwent R1 resection; anastomotic site recurrence with kidney infiltration and encasement of upper ureter. Systemic recurrence was in the form of peritoneal disease.

\section{Summary of short-term outcomes}

Perioperative mortality was seen in 2 patients (7.4\%). R0 resection was performed in $26 / 27$ patients (96.3\%). Final histopathology revealed adjacent organ infiltration in 19 cases (pT4b, 70.4\%). All surviving patients received adjuvant chemotherapy.

\section{DISCUSSION}

It may be difficult to differentiate intraoperatively malignant infiltration of colorectal tumor from inflammatory adhesion. Therefore, the standard management protocol mandates en bloc resection of the tumor along with the adjacent organ. In our study, malignant infiltration was histopathologically confirmed in $70.4 \%$ of patients. Nishikawa et al. [3] reported adjacent organ infiltration in $60.9 \%$ of patients, Eveno et al. [4] reported adjacent organ infiltration in $64.5 \%$, and Gebhardt et al. [5] reported adjacent organ infiltration in $55 \%$. Few previous studies demonstrated adhesions between tumor and other organs harbor malignant cells in $25 \%$ to $40 \%$ of cases, which are lower rates compared with our study $[2,6,7]$.

Local recurrence rates are also reported to be higher when the adjacent organs were dissected from the tumor than when en bloc resection is performed [8]. R0 resection is known to be one of the most important prognostic factors in the management of locally advanced colorectal cancer [9]. The rate of $\mathrm{R} 0$ resection, as reported in literature varies between $40 \%$ and $90 \%$ [10].

In our study, R0 resection was performed in $96.3 \%$ of patients; $18 / 18$ patients with colonic primary and $8 / 9$ patients with rectal primary. Eveno et al. [4] reported there were $89.5 \%$ of R0 resections in patients with clinical T4 colorectal cancer, but also reported $\mathrm{R} 1$ resections were due to invasion of the resection margin

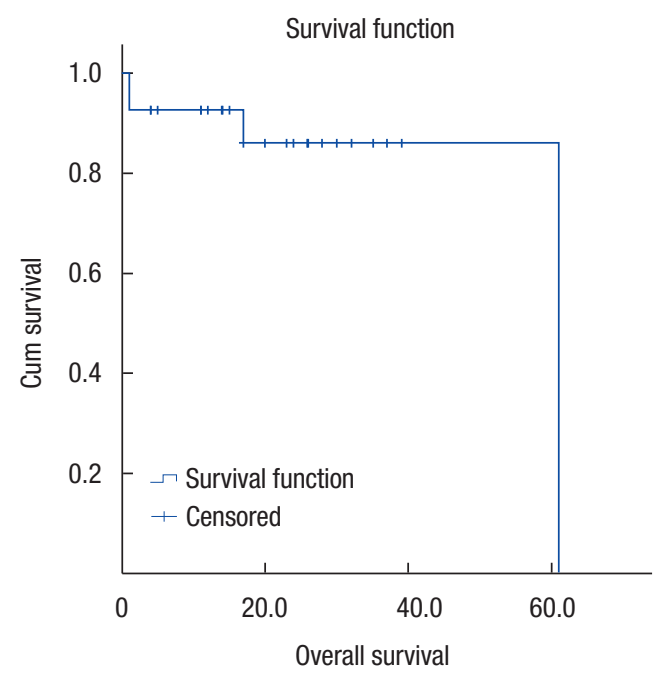

Fig. 1. Kaplan-Meier curve of overall survival.

of an adjacent organ in $5.2 \%$ of patients and due to invasion of the circumferential resection margin in $9.9 \%$ of patients and one R2 resection due to a large rectal cancer. In a retrospective study, Derici et al. [11] reported there were $75.4 \%$ of $\mathrm{R} 0$ resections in rectal cancer patients with macroscopically direct invasion to adjacent organs or structures and $82.8 \% \mathrm{R} 0$ resection in patients who received neoadjuvant chemoradiotherapy.

Three patients in our series developed recurrence. Circumferential resection margin was positive in 1 lower rectal cancer patient who developed recurrence in 11 months with pelvic sidewall infiltration, colovaginal fistula and died 17 months after completing treatment. One patient with splenic flexure growth who underwent curative treatment developed anastomotic site recurrence after 18 months with frank infiltration into the left kidney and encasement of the left upper ureter. The patient underwent curative surgery for recurrent disease with left nephroureterectomy. One patient developed peritoneal recurrence after 14 months with a peritoneal carcinomatosis index of 6 for which secondary cytoreduction was performed. One patient was lost to follow up.

The oncologic outcomes of the multivisceral resections are reported as overall survival rates of $30 \%$ to $53 \%[2,4,11]$. We could not demonstrate a distinct survival advantage due to the limited number of patients and the relatively short follow-up period. Kaplan-Meier curve of overall survival is shown in Fig. 1.

Multivisceral resection has been shown to be an independent factor for postoperative complications and perioperative mortality [12]. Studies report postoperative morbidity and mortality rates after multivisceral resection in the range from $28.0 \%$ to $43.7 \%[2,3,7,11,13-15]$ and $\geq 13 \%[4,6,11,16]$, respectively. In our study postoperative complications were identified in $25.9 \%$ patients. There were 2 mortalities (7.4\%) in our study. The adjacent organs resected are shown in Table 2 and Fig. 2.

There are some limitations of our study. First, it is a single-center 

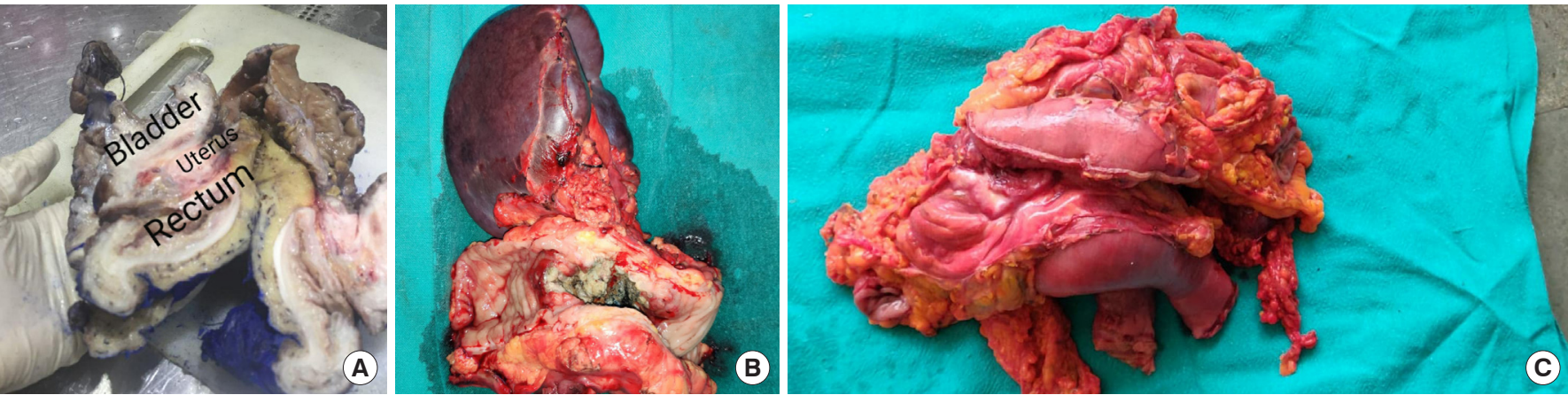

Fig. 2. (A) Pelvic exenteration. (B) Left hemicolectomy with splenectomy and distal pancreatectomy. (C) Transverse colectomy with sleeve gastrectomy and segmental jejunal resection.

study with a limited number of patients. Second, the follow-up period is relatively short with proportionately more cases being done over the past 12 months.

In conclusion, complete removal of all gross and microscopic disease remains the key to achieve long term outcomes in locally advanced colorectal cancer. Multivisceral resection can be performed at high volume centers with acceptable morbidity and mortality rates. Most of the recurrences occur within 2 years of completing treatment. Hence, meticulous follow-up is of paramount importance during this period. Longer follow-up is needed for survival data to mature.

\section{CONFLICT OF INTEREST}

No potential conflict of interest relevant to this article was reported.

\section{REFERENCES}

1. Jemal A, Center MM, DeSantis C, Ward EM. Global patterns of cancer incidence and mortality rates and trends. Cancer Epidemiol Biomarkers Prev 2010;19:1893-907.

2. Sökmen S, Terzi C, Unek T, Alanyali H, Füzün M. Multivisceral resections for primary advanced rectal cancer. Int J Colorectal Dis 1999;14:282-5.

3. Nishikawa T, Ishihara S, Emoto S, Kaneko M, Murono K, Sasaki K, et al. Multivisceral resections for locally advanced colorectal cancer after preoperative treatment. Mol Clin Oncol 2018;8:493-8.

4. Eveno C, Lefevre JH, Svrcek M, Bennis M, Chafai N, Tiret E, et al. Oncologic results after multivisceral resection of clinical T4 tumors. Surgery 2014;156:669-75.

5. Gebhardt C, Meyer W, Ruckriegel S, Meier U. Multivisceral resection of advanced colorectal carcinoma. Langenbecks Arch Surg 1999;384:194-9.

6. Gezen C, Kement M, Altuntas YE, Okkabaz N, Seker M, Vural S, et al. Results after multivisceral resections of locally advanced colorectal cancers: an analysis on clinical and pathological T4 tu- mors. World J Surg Oncol 2012;10:39.

7. Lehnert T, Methner M, Pollok A, Schaible A, Hinz U, Herfarth C. Multivisceral resection for locally advanced primary colon and rectal cancer: an analysis of prognostic factors in 201 patients. Ann Surg 2002;235:217-25.

8. Hunter JA, Ryan JA Jr, Schultz P. En bloc resection of colon cancer adherent to other organs. Am J Surg 1987;154:67-71.

9. Smith JD, Nash GM, Weiser MR, Temple LK, Guillem JG, Paty PB. Multivisceral resections for rectal cancer. Br J Surg 2012;99:113743.

10. Hallet J, Zih FS, Lemke M, Milot L, Smith AJ, Wong CS. Neo-adjuvant chemoradiotherapy and multivisceral resection to optimize R0 resection of locally recurrent adherent colon cancer. Eur J Surg Oncol 2014;40:706-12.

11. Derici H, Unalp HR, Kamer E, Bozdag AD, Tansug T, Nazli O, et al. Multivisceral resections for locally advanced rectal cancer. Colorectal Dis 2008;10:453-9.

12. Nakafusa Y, Tanaka T, Tanaka M, Kitajima Y, Sato S, Miyazaki K. Comparison of multivisceral resection and standard operation for locally advanced colorectal cancer: analysis of prognostic factors for short-term and long-term outcome. Dis Colon Rectum 2004;47:2055-63.

13. Nagasue Y, Akiyoshi T, Ueno M, Fukunaga Y, Nagayama S, Fujimoto $\mathrm{Y}$, et al. Laparoscopic versus open multivisceral resection for primary colorectal cancer: comparison of perioperative outcomes. J Gastrointest Surg 2013;17:1299-305.

14. Kim KY, Hwang DW, Park YK, Lee HS. A single surgeon's experience with 54 consecutive cases of multivisceral resection for locally advanced primary colorectal cancer: can the laparoscopic approach be performed safely? Surg Endosc 2012;26:493-500.

15. Shukla PJ, Trencheva K, Merchant C, Maggiori L, Michelassi F, Sonoda T, et al. Laparoscopic resection of $t 4$ colon cancers: is it feasible? Dis Colon Rectum 2015;58:25-31.

16. Eisenberg SB, Kraybill WG, Lopez MJ. Long-term results of surgical resection of locally advanced colorectal carcinoma. Surgery 1990;108:779-85. 